Revista da Universidade Estadual de Alagoas/UNEAL e-ISSN 2318-454X, Ano 12, Vol. 12 (3), julho/outubro (2020).

\title{
Bioactivity of Neem oil in leafhopper nymphs Empoasca kraemeri (Hemiptera: Cicadellidae)
}

\section{Bioatividade do óleo de Neem em ninfas de cigarrinha Empoasca kraemeri (Hemiptera: Cicadellidae)}

\author{
Maria Jéssica dos Santos CABRAL ${ }^{1}$; Rodrigo Almeida PINHEIRO². \\ ${ }^{1}$ Mestranda em Produção Vegetal; Universidade Federal dos Vales do Jequitinhonha e Mucuri; Diamantina - \\ MG; \\ ${ }^{2}$ Mestrando em Produção Vegetal; Universidade Federal dos Vales do Jequitinhonha e Mucuri; Diamantina - \\ MG; E-mail: rodrigo6450@gmail.com. \\ *E-mail do autor principal: jessicacabral810@gmail.com.
}

Recebido em agosto de 2020 e aceito em outubro de 2020.

Resumo - A cigarrinha-verde Empoasca kraemeri (Ross e Moore, 1957) (Hemiptera: Cicadellidae), tem sido relatada como uma das principais pragas que atacam I. batatas. $\mathrm{O}$ Neem Azadirachta indica é uma alternativa viável no controle de insetos pragas, pois é um produto natural, não polui o meio ambiente e não causa resistência. Portanto, o presente trabalho teve como objetivo avaliar a bioatividade do óleo de Neem sobre ninfas de $E$. kraemeri. Para constatação do efeito folhas de Ipomea batatas foram pulverizados nas concentrações 6,$0 ; 3,0 ; 1,5 ; 0,75$ e 0,25 ul 1-1. Foram fornecidas a ninfas e as testemunhas foram pulverizadas com água destilada. O experimento foi mantido, em laboratório, a $25 \pm$ $2^{\circ} \mathrm{C}$; UR de $50 \pm 10 \%$ e fotofase de $12 \mathrm{~h}$. O período de incubação, para todos os tratamentos inclusive as testemunhas, foi de três dias. As concentrações 1,5, 3,0 e 6,0 ul foram as que atingiram mortalidade de $92 \%, 100 \%$ e $100 \%$ nas ninfas de E. kraemeri, demonstrando o potencial da Azadirachta indica para controle da cigarrinha. Portanto, conclui-se que, o óleo de Neem ocasionou significativa mortalidade sobre as ninfas de Cigarrinha E. kraemeri .

Palavras-chave: Concentrações. Extratos. Mortalidade.

Abstract - The green leafhopper Empoasca kraemeri (Ross and Moore, 1957) (Hemiptera:
Cicadellidae), has been reported as one of the main pests that attack I. potatoes. Neem
Azadirachta indica is a viable alternative in the control of insect pests, as it is a natural
product, does not pollute the environment and does not cause resistance. Therefore, the
present study aimed to evaluate the bioactivity of Neem oil on nymphs of E. kraemeri. To
verify the effect of leaves of Ipomea potatoes were sprayed at concentrations $6.0 ; 3.0 ; 1.5$;
0.75 and 0.25 ul $1-1$. Nymphs were supplied and the controls were sprayed with distilled
water. The experiment was maintained, in the laboratory, at $25 \pm 2{ }^{\circ} \mathrm{C} ; 50 \pm 10 \% \mathrm{RH}$ and 12
h photophase. The incubation period, for all treatments including controls, was three days.
Concentrations $1.5,3.0$ and 6.0 ul were those that reached $92 \%, 100 \%$ and $100 \%$ mortality in 
Revista da Universidade Estadual de Alagoas/UNEAL e-ISSN 2318-454X, Ano 12, Vol. 12 (3), julho/outubro (2020).

E. kraemeri nymphs, demonstrating the potential of Azadirachta indica for leafhopper control. Therefore, it is concluded that the Neem oil caused significant mortality on the nymphs of Cigarrinha E. kraemeri.

Keywords: Concentrations. Extracts. Mortality.

\section{Introdução}

A batata-doce (Ipomoea batatas) é uma hortaliça tuberosa bastante disseminada em todo o território brasileiro, caracterizando-se por sua rusticidade, baixo custo de produção, ampla adaptação a diferentes ambientes e por sua tolerância à seca (MAINO et al., 2019).

A batata-doce é um alimento importante por ser altamente nutritiva e ter grande concentração de vitamina A (PERES et al., 2020). I. batatas é uma erva trepadeira, suas flores afuniladas crescem em cachos em suas ramas e têm matizes de rosa ou violeta (BEVILAQUA et al., 2019).

A raiz da batata-doce é a parte comestível (SOUSA, et al., 2018). Cada planta produz entre quatro a dez raízes, geralmente com formato oval e com a extremidade pontuda (ANDRADE, et al., 2017). I.batatas é consumida de várias formas, suas folhas também podem ser consumidas, e tanto a raiz como as ramas são muito usadas para alimentar animais e para produção de biocombustíveis (CIP, 2019).

Empoasca kraemeri cigarrinha-verde (Ross e Moore, 1957) (Hemiptera: Cicadellidae), está sendo relatada como inseto praga que atacam I. batatas (OLIVEIRA et al., 2016). Os danos provocados por esta espécie é de ordem física, por penetrar seu estilete no floema da planta, ocasionando a desorganização e a destruição das células e dos vasos condutores de seiva (CARVALHO et al., 2016).

Ocasiona também a injeção de substâncias toxicológicas durante a alimentação (BRAGA, 1998). Altas infestações da praga levam a atrofiar as plantas, que passam a possuir as bordas dos folíolos viradas para baixo (BRAGA, 1998). Nós casos mais severos, ocorre o amarelecimento dos folíolos e posteriormente o secamento das folhas (MOURA et al., 2018). Para o controle desta praga é necessário procurar medidas alternativas que sejam adaptáveis e menos prejudiciosas, especialmente para os agricultores.

Uma dessas alternativas é árvore do neem (Azadirachta indica), de origem asiática, contém cerca de 135 compostos que podem ter ação contra insetos pragas (QUEIROZ et al ., 2020). Entre estes compostos, destaca-se a azadiractina A (AZA) (RODRIGUES et al., 2018). A concentração de oleo de neem pode atingir $40 \%$ no óleo extraído (QUEIROZ et al ., 2020). Em decorrência das suas características, o Neem pode ser uma alternativa viável no controle de pragas, pois é um produto natural, não polui o meio ambiente e não causa resistência (MORÁN et al., 2018).

As experiências realizadas com neem mostraram algumas propriedades acaricidas de seus extratos (COSTA et al., 2016). A árvore de Neem consorciada com a pastagem pode 
Revista da Universidade Estadual de Alagoas/UNEAL e-ISSN 2318-454X, Ano 12, Vol. 12 (3), julho/outubro (2020).

ser um método alternativo de manejo integrado de pragas (OLIVEIRA et al., 2018). Vários estudos foram realizados para desenvolver medidas de controle ambientalmente seguras contra esses tipos de pragas e tentativas foram feitas com o uso da é árvore de neem A. indica (PEIXOTO et al., 2017).

Para o controle de pragas alguns pesquisadores indicam a fase de ninfa que é fase mais sensível da praga onde está na transição para se torna adulto. Com base nisto, o presente trabalho teve como objetivo avaliar a bioatividade do óleo de Neem sobre ninfas E. kraemeri.

\section{Procedimentos metodológicos}

\section{Local e condições dos experimentos}

O experimento foi conduzido no Laboratório de Entomologia e em Casa de Vegetação no setor de Olericultura da Universidade Federal dos Vales do Jequitinhonha e MucuriUFVJM, em Diamantina, Minas Gerais (altitude $1.387 \mathrm{~m}, 18^{\circ} 10^{\prime} \mathrm{S}$ de latitude e $43^{\circ} 30^{\prime} \mathrm{W}$ de longitude). $\mathrm{O}$ experimento constou as seguintes etapas:

\section{Condução da cultura}

Plantas de batata-doce, Ipomoea batatas L. (Solanales: Convolvulaceae) foram cultivadas em casa de vegetação, as folhas dessas plantas foram utilizadas para a criação dos insetos e, também, nos bioensaios. Folhas de batata doce com aproximadamente 90 dias de idade receberam ninfas e adultos por folha de E. kraemeri.

\section{Criação da Cigarrinha: Empoasca kraemeri (Hemiptera: Cicadellidae)}

Os adultos foram mantidos em gaiola de madeira e tela de náilon de $6 \mathrm{~mm}(3,0$ x 3,0 x $0,50 \mathrm{~m}$ ). Para obtenção das posturas foram colocados no interior da gaiola pecíolos de batata doce imersos em vidros com água. Após a oviposição, recipientes de vidros contendo folhas com as posturas, foram colocados em gaiola de madeira para a obtenção das ninfas.

\section{Ação translaminar}

O delineamento utilizado foi inteiramente casualizado com seis tratamentos e cinco repetições. Os tratamentos foram compostos por cinco diluições do óleo de Neem comercial 
Revista da Universidade Estadual de Alagoas/UNEAL e-ISSN 2318-454X, Ano 12, Vol. 12 (3), julho/outubro (2020).

SEMPRE VERDE KILLER NEEM® (Duas doses a cima da comercial (1,5ul) e duas doses abaixo da comercial) em água destilada com teor inicial de azadiractina de $300 \mathrm{mg} . \mathrm{L}^{-1}$ e água destilada como controle. $\mathrm{O}$ extrato foi diluído em água destilada para obtenção das concentrações 6,$0 ; 3,0 ; 1,5 ; 0,75$ e 0,25 ul $1-1$.

Em seguida, procedeu-se a pulverização nos folíolos de batata doce em cada concentração, conforme os procedimentos recomendados pelo Grupo Internacional das Associações Nacionais de Fabricantes de Produtos Agroquímicos (GIFAP). As testemunhas foram constituídas por pulverização dos folíolos com água destilada.

Avaliou-se diariamente a obtenção de morte e sobrevivência das ninfas. O experimento foi avaliado em 24, 48 e 72 horas. O experimento foi mantido, em laboratório, a $25 \pm 2{ }^{\circ} \mathrm{C}$; UR de $50 \pm 10 \%$ e fotofase de $12 \mathrm{~h}$. Todos os parâmetros foram submetidos à análise de variância, sendo a comparação entre médias dos tratamentos feita pelo teste de Tukey $(\mathrm{P} \leq 0,05)$, através do programa computacional SISVAR (Versão 5.6).

\section{Resultados e discussão}

Os resultados da pesquisa demonstraram efeito significativo para o teste de Tukey a $5 \%$ de probabilidade, foi observado diferenças entre os tratamentos aplicados nas ninfas de Empoasca kraemeri na superfície das folhas com um coeficiente de variação de $12.12 \%$ (Tabela 1). A eficiência de controle de derivados de Neem também foi relatada sobre outras espécies de insetos pragas, como, L. huidobrensis no feijoeiro (Phaseolus vulgaris L.) (DEQUECH et al., 2010; e L. trifolii no feijoeiro e tomateiro (RAI et al., 2013; YILDIRIM, 2012).

Tabela 1 - Tabela da Análise de Variância para o experimento com 6 diferentes concentrações do extrato de Azadirachta indica em ninfas e adultos de Empoasca kraemeri

\begin{tabular}{llcccc}
\hline FV & GL & SQ & QM & Fc & Pr>Fc \\
\hline Tratamento & 5 & 91.1 & 18.2 & 99.3 & 0.0000 \\
Resíduo & 24 & 4.4 & 0.19 & & \\
Total & 29 & 95.5 & & & \\
\hline CV\% & & & $\mathbf{1 2 . 1 2}$ & &
\end{tabular}

Fonte: Acervo do autor.

Observou-se uma mortalidade significativa de ninfas de acordo com as concentrações. Maiores concentrações obtiveram médias maiores na taxa de mortalidade (1,5; 3,0 e 6,0 ul-1) com mortalidades de $92 \%, 100 \%$ e $100 \%$, respectivamente para as ninfas (Tabela 2). A concentração comercial recomendada obteve uma média de mortalidade altamente significativa, ou seja, podendo ser utilizada de acordo com a descrição que vem no produto. 
Revista da Universidade Estadual de Alagoas/UNEAL e-ISSN 2318-454X, Ano 12, Vol. 12 (3), julho/outubro (2020).

Foi observada também uma igualdade na taxa de mortalidade com as duas ultimas doses, ou seja, tanto faz usar 3,0 ul ou 6,0 ul as duas conseguiram 100\% de mortalidade (Tabela 2).

De acordo com estudos realizados por Rai et al., (2013), onde ele mostrou que a ação do óleo de Neem pode variar de acordo com o aumento das dosagens utilizadas. Há inúmeros registros na literatura que apontam alterações na dose comercial com comparação as doses mais altas na mortalidade no ciclo de vida de diferentes espécies de insetos (ALVARENGA et al., 2012; HOSSAIN et al., 2008; HOSSAIN, 2006; SCHMUTTERER, 1990; SILVA, 2009; WEINTRAUB, 1997).

Tabela 2. Médias da mortalidade diária de ninfas e adultos de Empoasca kraemeri com diferentes concentrações do extrato de Azadirachta indica a 5\% de probabilidade.

\begin{tabular}{cc}
\hline Tratamentos & Médias \\
\hline T1 & $0.0 \mathrm{c}$ \\
T2 & $3.0 \mathrm{~b}$ \\
T3 & $3.6 \mathrm{~b}$ \\
T4 & $4.6 \mathrm{a}$ \\
T5 & $5.0 \mathrm{a}$ \\
T6 & $5.0 \mathrm{a}$ \\
\hline Média geral & $\mathbf{3 . 5}$
\end{tabular}

Médias seguidas pela mesma letra, nas colunas, não diferem entre si pelo teste Tukey $(\mathrm{P} \leq 0,05)$. Fonte: Acervo do autor.

De acordo com a linha de tendência de aumento na (Figura 1), é possível notar com nitidez o aumento da mortalidade das ninfas de acordo com o aumento das dosagens de óleo de Neem. Neste caso, a dose abaixo da comercial provocou índice de mortalidade maior que 50\% nas ninfas de E. kraemeri, chegando a quase $80 \%$ de mortalidade. 
Revista da Universidade Estadual de Alagoas/UNEAL e-ISSN 2318-454X, Ano 12, Vol. 12 (3), julho/outubro (2020).

Figura 1. Mortalidade de ninfas de Empoasca kraemeri em função das concentrações do óleos de Azadirachta indica em folíolos de Ipomea batatas.

Mortalidade de ninfas Empoasca kraemeri

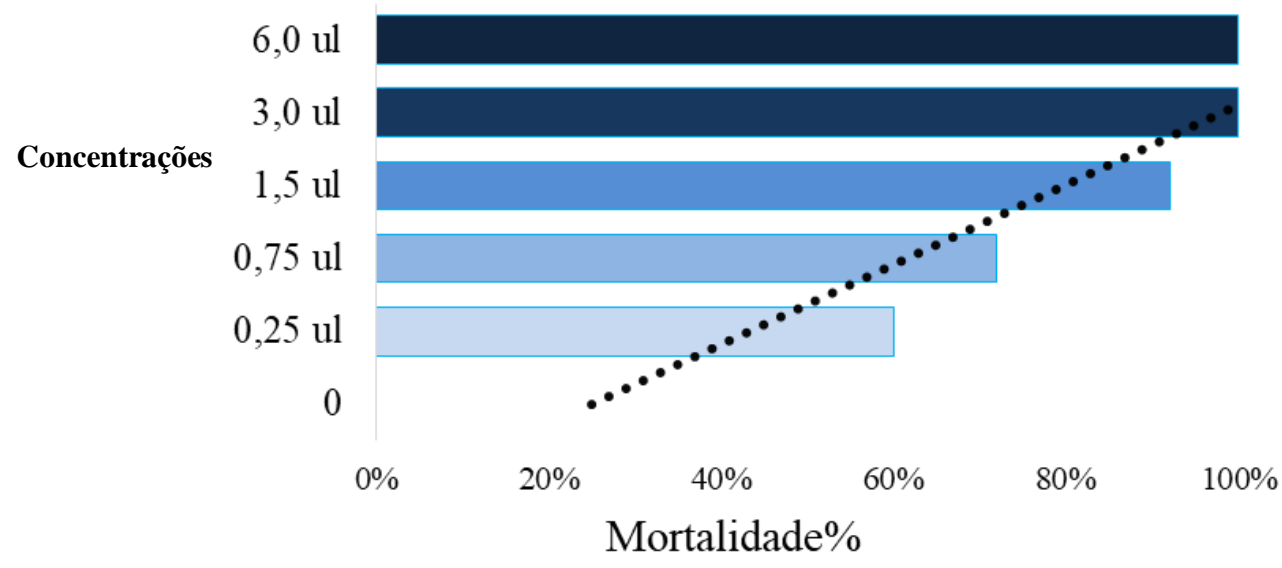

Fonte: Acervo do autor.

No tratamento-testemunha, por sua vez, todas as cigarrinhas se encontraram vivas (Figura 1). Resultados semelhantes foram observados sob o efeito de contato tópico, quanto aos valores de mortalidade de $S$. zeamais foram significativamente superior com a aplicação do óleo de Neem, em todas as concentrações mais altas avaliadas (COSTA et al., 2016).

\section{Conclusão}

Portanto, os tratamentos à base de óleo de A. Indica foram significativos para o número de mortes de ninfas de E. kraemeri nas maiores concentrações , 1,5 ul, 3,0 ul e 6,0 ul, podendo assim ser recomendados como alternativa no manejo desta praga, uma vez que, ao diminuir a quantidade de ninfas na plantação irá diminuir a quantidade de insetos na planta, minimizando também os danos diretos e os indiretos, como também a transmissão de doenças virais.

\section{Conflito de interesses}

Os autores deste manuscrito não declararam conflitos de interesse. 


\section{Referências}

ALVARENGA, C. D. et al. Toxicity of neem (Azadirachta indica) seed cake to larvae of the Mediterranean fruit fly, Ceratitis capitata (Diptera: Tephritidae), and its parasitoid, Diachasmimorpha longicaudata (Hymenoptera: Braconidae). Florida Entomologist, v. 95, n. 1, p. 57-62, 2012.

ANDRADE, E. K. V. de et al. Dissimilaridade genética entre genótipos de batata-doce por meio de descritores morfológicos e moleculares. Acta Scientiarum. Agronomy, v. 39, n. 4, p. 447-455, 2017.

BEVILAQUA, L.K.S. et al. Características morfológicas e produtivas de clones de batata doce. Embrapa Semiárido-Artigo em periódico indexado (ALICE), 2019.

BRAGA, J.S. Estimativa do risco da cigarrinha verde da vinha. Relat. Final Curso Eng. Agrón. UTL/ISA, Lisboa (1998).

CARVALHO, D. P. Population fluctuation of Empoasca sp.(Hemiptera: Cicadellidae) in a physic nut crop in Mato Grosso do Sul. Acta Biológica Colombiana, v. 21, n. 1, p. 81-85, 2016.

COSTA, E. M. et al. Aqueous extract of neem seeds in the control of Liriomyza sativae (Diptera: Agromyzidae) in the melon. Revista Ciência Agronômica, v. 47, n. 2, p. 401-406, 2016.

COSTA, E. M. et al. Aqueous extract of neem seeds in the control of Liriomyza sativae (Diptera: Agromyzidae) in the melon. Revista Ciência Agronômica, v. 47, n. 2, p. 401-406, 2016.

DEQUECH, S. T. B. et al. Inseticidas botânicos sobre Liriomyza huidobrensis Blanchard (Diptera: Agromyzidae) e seus parasitoides em feijão-de-vagem cultivado em estufa.

Biotemas, v. 23, n. 2, p. 37-43, 2010.

DIMETRY, N. Z. et al. Evaluation of two neem seed kernel extracts against Liriomyza trifolii (Burg.) (Dip. Agromyzidae). Anzeiger Für Schädlingskunde, Pflanzenschutz, Umweltschutz, v. 68, n. 2, p. 39-41, 1995.

HOSSAIN, M. B. et al. Effects of soil application of neem (NeemAzal ${ }^{\circledR}$ - U) on different life stages of Liriomyza sativae (Diptera: Agromyzidae) on tomato in the humid tropic. Journal of Plant Diseases and Protection, v. 115, n. 2, p. 80-87, 2008.

HOSSAIN, M. B. et al. Effects of a neembased insecticide on different immature life stages of the leafminer Liriomyza sativae on tomato. Phytoparasitica, v. 34, n. 4, p. 360-369, 2006. 
Revista da Universidade Estadual de Alagoas/UNEAL e-ISSN 2318-454X, Ano 12, Vol. 12 (3), julho/outubro (2020).

International Potato Center (CIP), fatos sobre a batata doce: um compêndio de figuras-chave e análise para 33 importantes países produtores de batata-doce, produção, uso, Consumo e Uso de Alimentos, Centro Internacional de Batata (CIP), Lima, Peru, 2019.

MAINO, S. C. et al. Batata-doce (Ipomoea batatas) dentro do contexto de culturas energéticas, uma revisão. Revista Brasileira de Energias Renováveis, v. 8, n. 4, 2019.

MORÁN, C. S. et al. Uso de bioinsecticida a base de neem Azadirachta indica para el manejo de saltahoja en agroecosistema de caña de azúcar, Guayas, Ecuador. Manglar, v. 14, n. 1, p. 73-83, 2018.

MOURA, M. F. et al. Sequential sampling plans and economic injury levels for Empoasca kraemeri on common bean crops at different technological levels. Pest management science, v. 74, n. 2, p. 398-405, 2018

OLIVEIRA, E. L. et al. Monitoramento e identificação dos insetos pragas na cultura do maracujazeiro (Passiflora spp.) para implementação do manejo integrado de pragas. Cadernos de Agroecologia, v. 13, n. 1, 2018.

OLIVEIRA, M. D. G. et al. Spatial distribution of Empoasca kraemeri (Hemiptera:

Cicadellidae) adults in physic nut Jatropha curcas L. Entomotropica, v. 31, n. 29, p. 237-243, 2016.

PEIXOTO, M. L. et al. Associação entre genótipos de feijoeiro, silício e nim no controle da mosca-branca Bemisia tabaci (Gennadius, 1889) biótipo B (Hemiptera: Aleyrodidae). Revista Ceres, v. 64, n. 4, p. 376-383, 2017.

PERES, A. P. et al. Avaliação nutricional de colaboradores de um hotel convention Curitiba/PR/Nutritional evaluation of contributors of a hotel convention Curitiba/PR. Brazilian Journal of Development, v. 6, n. 6, p. 34418-34430, 2020.

QUEIROZ, T. et al. EXTRATOS E ÓLEOS ESSENCIAIS COMO ALTERNATIVA NO CONTROLE DE Sclerotinia sclerotiorum E Sclerotium rolfsii ISOLADOS DE SOJA (Glycine max L.). Revista em Agronegócios e Meio Ambiente, v. 13, n. 2, 2020.

RAI, D.S. et al. Efficacy of insecticides against american serpentine leafminer, Liriomyza trifolii (Burgess) on tomato crop in N-W region of Uttar Pradesh, India. International Journal of Horticulture, v. 5, p. 19-21, 2013.

RODRIGUES, L. C. et al. Ação repelente de óleo de neem durante o cultivo de duas espécies de physalis. Anais Seminário de Iniciação Científica, n. 20, 2018. 
Revista da Universidade Estadual de Alagoas/UNEAL e-ISSN 2318-454X, Ano 12, Vol. 12 (3), julho/outubro (2020).

SCHMUTTERER, H. Properties and potential of natural pesticides from the neem tree, Azadiractha indica. Annual Review of Entomology, v. 35, p. 271-297, 1990.

SILVA, A. B. et al. Atividade inseticida do nim (Azadiractha indica A. Juss). Revista verde de Agroecologia e Desenvolvimento Sustentável, v. 4, p. 7-15, 2009.

SOUSA, L.B.T. et al. Biscoitos elaborados com batata-doce de polpa alaranjada: avaliação físico-química, microbiológica e morfológica. Embrapa Agroindústria de AlimentosArtigo em periódico indexado (ALICE), 2018.

SOUZA XAVIER, et al. Comportamento de genótipos de feijão-caupi (Vigna unguiculata L. Walp., Fabaceae) sob infestação de Empoasca sp.(Hemiptera: Cicadellidae) e Liriomyza sp.(Diptera: Agromyzidae) em Redenção, Ceará, Brasil. Paubrasilia, v. 3, n. 1, p. 18-23, 2020

WEINTRAUB, P. G.; HOROWITZ, A. R.; Systemic Effects of a neem insecticide on Liriomyza huidobrensis larvae. Phytoparasitica, v. 25, n. 4, p. 283-289, 1997.

YILDIRIM, E. M.; BASPINAR, H. Effects of neem on Liriomyza trifolii (Burgess) (Diptera: Agromyzidae) and its parasitoids on tomato greenhouse. Journal of Food, Agriculture \& Environment, v. 10, n. 1, p. 381-384, 2012. 